\title{
A Case of Acute Coronary Artery Occlusion Associated with Very Rapid Onset Heparin-induced Thrombosis without Thrombocytopenia
}

\author{
Tatsuya Hondo ${ }^{1}$, Hiroya Matsumura ${ }^{1}$, Keiji Matsuda ${ }^{1}$, Akimichi Iwamoto ${ }^{1}$, \\ Shin Eno ${ }^{1}$ and Mitsuru Kimura ${ }^{2}$
}

\begin{abstract}
An 81-year-old woman was admitted to our hospital with acute heart failure. A coronary stent was implanted in the left circumflex artery (LCX) on day 3, and elective percutaneous coronary intervention for stenosis in the left anterior descending artery (LAD) was planned for day 10. However, 15 minutes after heparin administration, sudden thrombotic occlusion occurred first in the LAD and then in the LCX. Although anti-platelet factor 4/heparin antibody positivity was detected, there was no significant decrease in platelet counts. This suggests that in the event of unexplained thrombosis, heparin-induced thrombosis should be suspected irrespective of platelet counts and early onset.
\end{abstract}

Key words: heparin-induced thrombocytopenia and thrombosis syndrome, rapid onset, percutaneous coronary intervention

(Intern Med 51: 607-611, 2012)

(DOI: 10.2169/internalmedicine.51.6398)

\section{Introduction}

Heparin-induced thrombocytopenia and thrombosis syndrome (HITTS) is a drug-induced, anti-platelet factor 4/ heparin antibody-mediated disorder frequently associated with venous and/or arterial thromboembolic complications. Usually, symptoms develop 5-10 days following administration of heparin (typical onset type). However, in some patients with previous heparin exposure, symptoms develop within 24 hours (rapid onset type) (1). We describe a case of very rapid onset HITTS complicated by occlusion in two branches of the coronary artery. This phenomenon occurred 15 minutes after heparin readministration for an elective percutaneous coronary intervention (PCI). Anti-platelet factor 4/ heparin antibody positivity was detected; however, platelet counts were normal.

\section{Case Report}

An 81-year-old woman was admitted to our hospital be- cause of dyspnea. Her blood pressure was 140/80 $\mathrm{mmHg}$, and her regular pulse was 100 beats per minute. The level of oxygen saturation was approximately $80 \%$ during the administration of $10 \mathrm{~L} / \mathrm{min}$ oxygen via a mask. Chest X-ray revealed pulmonary edema. An electrocardiogram showed ST depression in leads V2-V5, but ST elevation was not detected in any lead. She was diagnosed as having acute heart failure, and treated using an artificial respirator and diuretics. On day 3 of hospitalization, emergency cardiac catheterization was performed because of acute lateral myocardial infarction with ST elevation in leads I and aVL. Coronary angiograms showed $99 \%$ stenosis of the left circumflex artery (LCX) and 90\% stenosis of the left anterior descending artery (LAD) (Fig. 1). Therefore, a coronary stent (XIENCE V $2.5 \times 23 \mathrm{~mm}$, Abbott) was implanted into segment 12 of the LCX from segment 11 using the slip through technique (2). The stent was smoothly delivered with the simultaneous deflation of a balloon positioned parallel to the stent (Fig. 2). Heparin was continuously administered till day 5 during the operation of an intra-aortic balloon pumping.

Elective PCI of the LAD lesion was planned for day 10.

${ }^{1}$ Department of Cardiology, Chugoku Rosai Hospital, Japan and ${ }^{2}$ Clinical Laboratory, Chugoku Rosai Hospital, Japan Received for publication August 16, 2011; Accepted for publication November 17, 2011

Correspondence to Dr. Tatsuya Hondo, hondo@chugokuh.rofuku.go.jp 

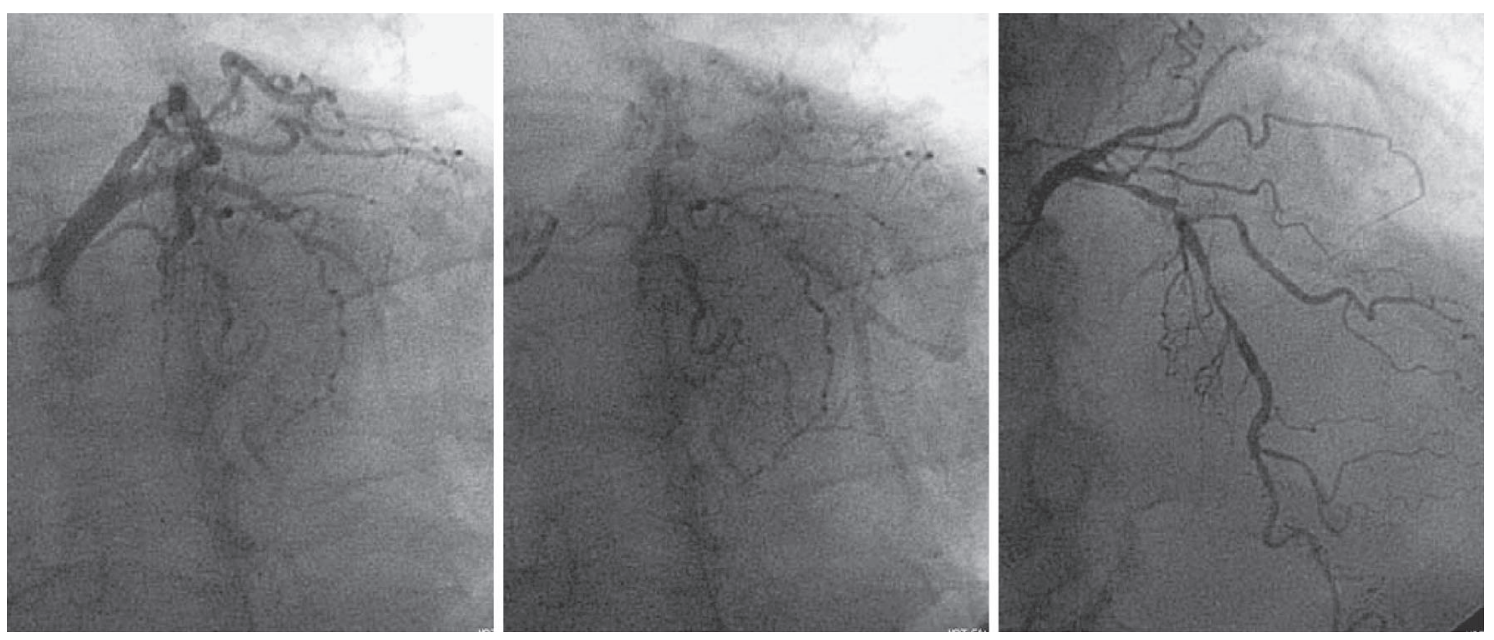

Figure 1. Coronary angiograms performed on day 3. The filling (left panel) and washout phases (middle panel) of the angiograms show $99 \%$ stenosis of the mid left circumflex artery (straight caudal view). The right panel shows $\mathbf{9 0 \%}$ stenosis of the left anterior descending artery (straight cranial view).

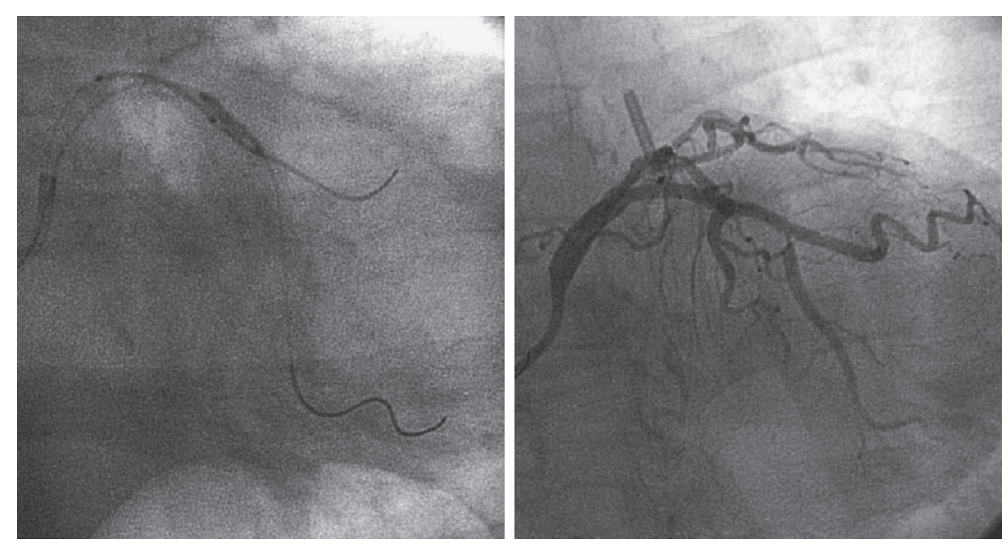

Figure 2. Percutaneous coronary intervention performed on day 3. A coronary stent was implanted in the left circumflex artery segment.

Intravenous bolus dose of 5,000 units of heparin was administered prior to the PCI procedure. However, 15 minutes later, the patient entered a state of shock. Coronary angiograms showed that the LAD was occluded at the proximal site. Occlusion of the LCX also subsequently occurred during the handling of the PCI guidewire (Fig. 3). Moreover, a thrombotic occlusion occurred inside the guide catheter. HITTS was immediately suspected because the sudden appearance of thrombosis could not be explained by coronary artery lesions, and all of the heparinized saline was immediately discarded. PCI was resumed using another guide catheter with an intra-aortic balloon pumping. Ten mg of argatroban was dissolved in $100 \mathrm{~mL}$ saline, and $10 \mathrm{~mL}$ of this solution was injected three times into the coronary artery while the remaining solution was intravenously administered. The thrombus in the LCX was aspirated via an aspiration catheter, and the flow was recovered. However, the aspiration catheter did not pass through the LAD lesion. Three stents (MINI VISION $2.5 \times 18 \mathrm{~mm}$ and $2.5 \times 23 \mathrm{~mm}$; Abbott Vascular, Duraflex $3.0 \times 14 \mathrm{~mm}$; Goodman) were then im- planted into the LAD from the periphery after balloon dilation. Postprocedural angiograms 20 minutes after completion of PCI revealed good flow and disappearance of the thrombi (Fig. 4).

After PCI, argatroban $0.7 \mu \mathrm{g} / \mathrm{kg} / \mathrm{min}$ was administered via continuous intravenous infusion. Oral administration of aspirin $81 \mathrm{mg}$ and clopidogrel $75 \mathrm{mg}$ was continued after admission. However, since there is no established method for assessing the efficacy of antiplatelet agents, cilostazol 200 mg was also administered in combination with aspirin and clopidogrel on the basis of the results of platelet aggregation test (3). Follow-up coronary angiograms performed on the following day and two days later under continuous infusion of argatroban and without heparin revealed no thrombus in the LAD or LCX.

The platelet counts were $204,000 / \mu \mathrm{L}$ on arrival, 258,000/ $\mu \mathrm{L}$ before elective PCI, $240,000 / \mu \mathrm{L}$ at the onset of HITTS, and $259,000 / \mu \mathrm{L}$ on the following day. Although the lowest value recorded was $169,000 / \mu \mathrm{L}$ on day 14 (4 days after the onset of HITTS), this was not a significant reduction 

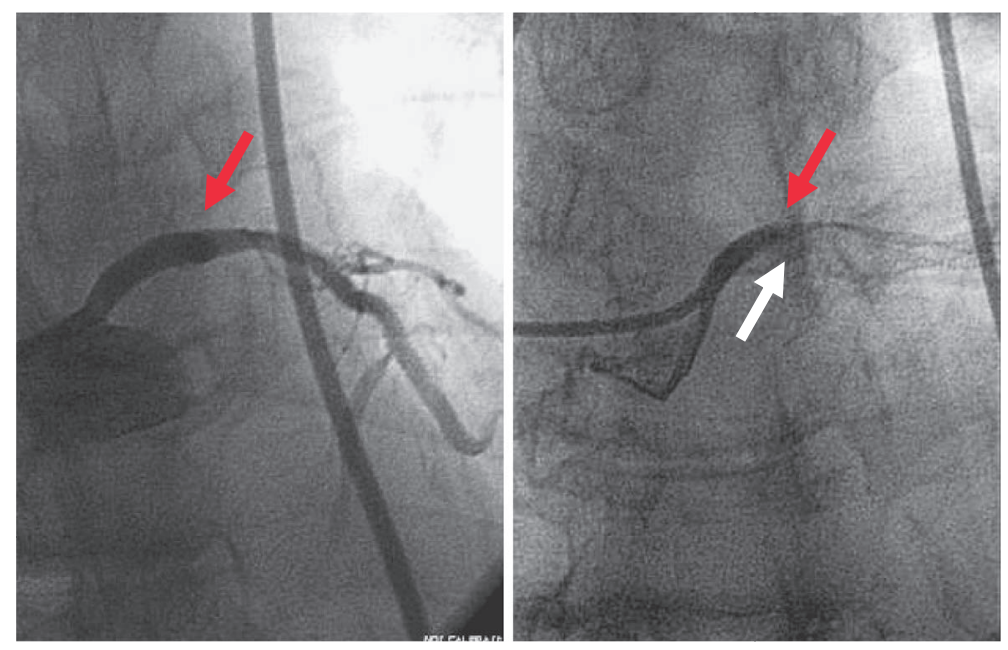

Figure 3. Elective percutaneous coronary intervention (PCI) of the left anterior descending artery was planned for day 10. A coronary angiogram 15 minutes after heparin administration revealed an occlusion of the left anterior descending artery (red arrow). Occlusion of the left circumflex artery also subsequently occurred (white arrow, right panel).
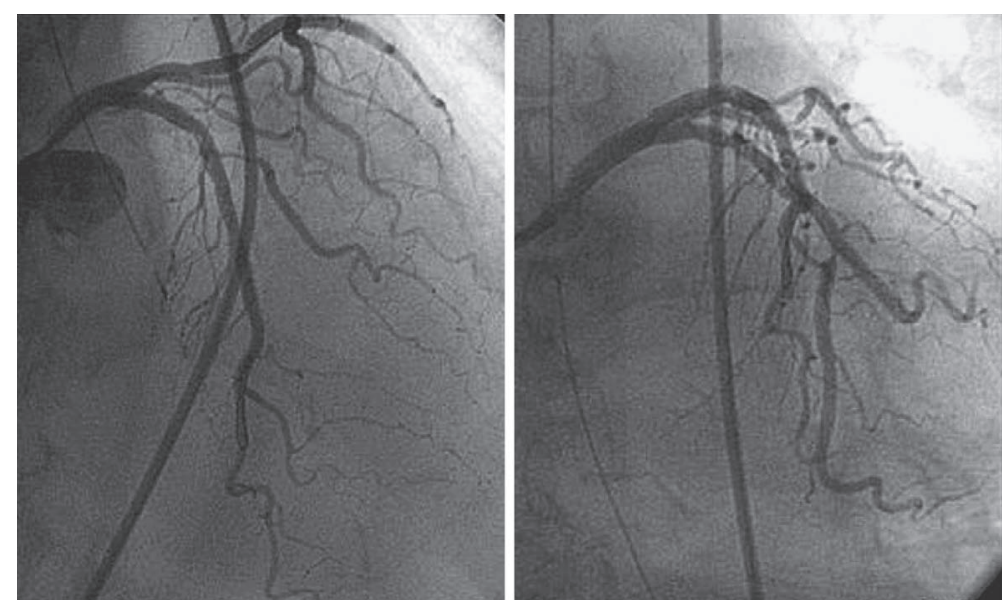

Figure 4. Coronary angiogram performed after percutaneous coronary intervention on day 10. Argatroban was administered intra-coronary and intravenously. The thrombus in the LCX was aspirated via an aspiration catheter, and three coronary stents were implanted into the left anterior descending artery from the periphery. Postprocedural angiograms show good flow and the disappearance of the thrombi.

(>50\%) from baseline (Fig. 5). An enzyme linked immunosorbent assay submitted on day 11 revealed anti-platelet factor 4/heparin antibody positivity. A heparin-provocation platelet aggregation test was performed on day 18, and an obvious increase in platelet aggregation was observed when the patient's serum was added to heparin-infused healthy platelet concentrates. The patient was discharged on day 48 following a period of rehabilitation.

\section{Discussion}

HITTS is usually complicated by anti-platelet factor 4/ heparin antibody-associated thromboembolism. Thrombosis in HITTS patients can occur at both intra-venous and intraarterial sites. Clinical background is considered to be associated with thrombus formation sites; venous thrombosis may be associated with surgery and arterial thrombosis with cardiovascular disease $(4,5)$. Symptoms of HITTS usually develop 5-10 days following the initiation of heparin administration. However in some patients with previous heparin exposure, symptoms develop within 24 hours. Warkentin and Kelton (1) analyzed 243 HITTS patients and reported that $30 \%$ had the rapid onset type with a median onset time of 10.5 hours.

Among 52,647 cases in the global registry of acute coronary syndrome (GRACE) (6), the incidence of heparininduced thrombocytopenia is reportedly $0.3 \%$, that of glycoprotein IIb/IIIa-associated thrombocytopenia is $0.6 \%$, and that of thrombocytopenia due to other reasons is $0.7 \%$. Moreover, the anti-platelet factor 4/heparin antibody positivity rate in patients with acute coronary syndrome (ACS) is high. Matsuo et al (7) reported anti-platelet factor 4/heparin 


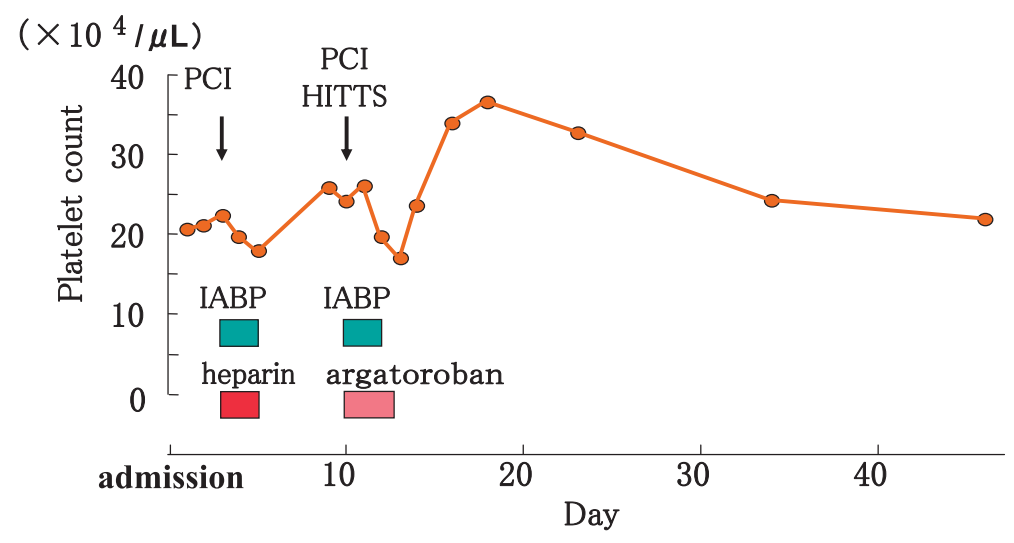

Figure 5. Platelet counts. The platelet counts were $258,000 / \mu \mathrm{L}$ before elective PCI, $240,000 / \mu \mathrm{L}$ at the onset of HITTS, and $259,000 / \mu \mathrm{L}$ on the following day. The lowest value recorded was $169,000 / \mu \mathrm{L}$ on day 14 (4 days after the onset of HITTS). HITTS: heparin-induced thrombocytopenia and thrombosis syndrome, PCI: percutaneous coronary intervention, IABP: intra-aortic balloon pumping.

antibody positivity in $22(8.7 \%)$ of 254 ACS patients who were treated with heparin. It has also been pointed out that this antibody can induce adverse events in the absence of thrombocytopenia as well (8).

For ACS patients, emergency PCI with heparin is often performed on admission, and frequently an elective PCI is also performed after the hemodynamics are confirmed stabilized. Anti-platelet factor 4/heparin antibody may be produced during this hemodynamically stable period, and thus the elective PCI may be performed during the high risk period (1, 9-11). Sakai et al (9) reported a case of inferior acute myocardial infarction where thrombi appeared in the LCX and LAD 40 minutes after readministration of heparin during elective PCI, which was performed 21 days after conservative treatment with heparin. Ziakas et al (10) described a case of unstable angina where thrombi appeared in the LCX and in the left main trunk of the coronary artery 50 minutes after readministration of heparin during elective PCI. Mibiki et al (11) also reported a case of acute simultaneous thrombosis of three coronary vessels during elective PCI on day 17.

In these cases, thrombosis occurred rapidly during the PCI procedure, creating an emergency where there is no time for blood tests and consequently making the diagnosis of HITTS difficult. The condition could be misunderstood as being caused by an insufficient dose of heparin, and could even be exacerbated by additional heparin administration. In addition, it should also be noted that thrombi appeared in multivessel and/or the left main trunk in theses cases. Our patient also entered a state of shock and unconsciousness due to simultaneous occlusion of the LAD and LCX.

In such cases, administration of heparin should be discontinued as soon as HITTS is suspected. However, discontinuation of heparin alone is insufficient and the use of other anticoagulant is required (4). Argatroban is approved for the treatment of HITTS in Japan. However, in such rapid onset cases where thrombi have instantly occluded multiple coronary arteries, the standard dose of argatroban and the means of its administration remain unclear. Sakai et al (9) administered $2.5 \mathrm{mg}$ bolus intravenous injection and used continuous infusion. Mibiki et al (11) administered $10 \mathrm{mg}$ of intravenous argatroban. Suzuki et al (12) injected argatroban into the coronary artery of a patient with repeated coronary thrombosis (who did not have HITTS antibodies). We also intravenously administered argatroban which was dissolved in $100 \mathrm{~mL}$ of saline into the coronary artery, and this led to the disappearance of the thrombi.

Platelet counts of HITTS patients usually decrease to less than $150,000 / \mu \mathrm{L}$ or more than $50 \%$ reduction from baseline $(1,4)$. Significant thrombocytopenia did not occur in the present case. Although the patient's clinical condition was serious, the amount of thrombi in the coronary arteries might be small. Insignificant thrombocytopenia might be due to the limited activation of platelets in the coronary arteries. A few other cases of heparin-induced thrombosis without thrombocytopenia have been previously described (13-15). Kawashima et al (15) described a case of myocardial infarction that developed recurrent heparininduced stent thrombosis without thrombocytopenia after an emergency stenting into the left main trunk of the coronary artery. They pointed out the possibility of a delay in diagnosis due to the lack of thrombocytopenia. Thus, HITTS should be considered as a possible diagnosis irrespective of the absence of thrombocytopenia (4). In addition, thrombosis developed 15 minutes after heparin administration in the present case, which was very rapid compared with the cases reported by Warkentin and Kelton (1). The cause of very rapid onset is unknown. Although the incidence of HITTS might be infrequent because heparin is widely used, it could occur in the cardiac angiography room, intensive care unit, or any other ward. If unexplained thrombosis occurs in patients with a history of heparin treatment, HITTS should be suspected. 


\section{Conclusion}

We describe a case of very rapid onset type HITTS without thrombocytopenia, which occurred 15 minutes after heparin readministration. Two branches of the coronary artery were consequently occluded. PCI was succeeded by immediate discontinuation of heparin and administration of argatroban. If unexplained thrombosis occurs in patients with a history of heparin treatment, HITTS should be suspected irrespective of platelet counts and the time of onset following heparin readministration

The authors state that they have no Conflict of Interest (COI).

\section{References}

1. Warkentin TE, Kelton JG. Temporal aspects of heparin-induced thrombocytopenia. N Engl J Med 344: 1286-1292, 2001.

2. Hondo T, Matsuda K, Eno S, Kisaka T, Tominaga K. Successful delivery of sirolimus-eluting coronary stents (SES) using a "slip through technique": deflating parallel balloon leads SES to the lesion smoothly. Jpn J Interv Cardiol 22: 155-160, 2007 (in Japanese).

3. Sairaku A, Nakano Y, Eno S, et al. Platelet function measured using a whole blood aggregometer can predict bleeding events. J Atheroscler Thromb 18: 16-23, 2011.

4. Shantsila E, Lip GY, Chong BH. Heparin-induced thrombocytopenia. A contemporary clinical approach to diagnosis and management. Chest 135: 1651-1664, 2009.

5. Boshkov LK, Warkentin TE, Hayward CP, Andrew M, Kelton JG. Heparin-induced thrombocytopenia and thrombosis: clinical and laboratory studies. Br J Haematol 84: 322-328, 1993.
6. Gore JM, Spencer FA, Gurfinkel EP, et al. GRACE Investigators. Thrombocytopenia in patients with an acute coronary syndrome (from the Global Registry of Acute Coronary Events [GRACE]). Am J Cardiol 103: 175-180, 2009.

7. Matsuo T, Tomaru T, Kario K, Hirokawa T; HIT Research Group of Japan. Incidence of heparin-PF4 complex antibody formation and heparin-induced thrombocytopenia in acute coronary syndrome. Thromb Res 115: 475-481, 2005.

8. Stribling WK, Slaughter TF, Houle TT, Sane DC. Beyond the platelet count: heparin antibodies as independent risk predictors. Am Heart J 153: 900-906, 2007.

9. Sakai K, Oda H, Honsako A, et al. Obstinate thrombosis during percutaneous coronary intervention in a case with heparin-induced thrombocytopenia with thrombosis syndrome successfully treated by argatroban anticoagulant therapy. Catheter Cardiovasc Interv 59: 351-354, 2003.

10. Ziakas A, Gavrilidis S, Makris P, Louridas G. Acute left main occlusion during percutaneous coronary intervention associated with heparin induced thrombocytopenia with thrombosis syndrome. J Interv Cardiol 18: 139-144, 2005.

11. Mibiki $Y$, Kikuta $H$, Yamaguchi $T$, et al. Acute thrombosis of three coronary arteries induced by the anti-heparin PF4 antibody after additional heparin administration for PCI. Jpn J Interv Cardiol 23: 448-453, 2008 (in Japanese).

12. Suzuki A, Aizawa Y, Yoshimura H, Hara T. The effectiveness of intracoronary infusion of argatroban in case of acute myocardial infarction with recurrent coronary obstruction by thrombosis. CVIT Jpn Ed 1: 81-84, 2009 (in Japanese).

13. Hach-Wunderle V, Kainer K, Salzmann G, Müller-Berghaus G, Pötzsch B. Heparin-related thrombosis despite normal platelet counts in vascular surgery. Am J Surg 173: 117-119, 1997.

14. Alvarez GF, Bihari D, Collins D. Heparin-induced thrombosis with a normal platelet count. Crit Care Resusc 9: 51-53, 2007.

15. Kawashima O, Abe H, Aono G. A case of very severe acute coronary syndrome caused by acute left main stent thrombosis repeated 3 times by SAT: A case of HIT without thrombocytopenia. CVIT Jpn Ed 2: 205-210, 2010 (in Japanese).

(C) 2012 The Japanese Society of Internal Medicine http://www.naika.or.jp/imindex.html 